\author{
Yuriy BILAN, DSc, PhD \\ Department of Finance, Banking and Accountancy \\ Rzeszow University of Technology, Rzeszow, Poland \\ E-mail: y.bilan@prz.edu.pl \\ Grzegorz MENTEL, DSc, PhD (Corresponding author) \\ Department of Quantitative Methods \\ Rzeszow University of Technology, Rzeszow, Poland \\ E-mail: gmentel@prz.edu.pl \\ Dalia STREIMIKIENE, PhD \\ Lithuanian Institute of Agrarian Economics, Vilnius, Lithuania \\ E-mail: dalia.streimikiene@ knf.vu.lt \\ Beata SZETELA, PhD \\ Department of Quantitative Methods \\ Rzeszow University of Technology, Rzeszow, Poland \\ E-mail: beata@prz.edu.pl
}

\title{
WEATHER RISK MANAGEMENT IN THE WEATHER-VAR APPROACH. ASSUMPTIONS OF VALUE-AT-RISK MODELING
}

\begin{abstract}
In this paper, an attempt is made to implement market-based risk measures in the process of weather risk management. A weather-VaR plays a significant role in the risk evaluation of non-extreme weather events and the process of its management, even in terms of weather derivatives. The innovative nature of the work results from the approach to model the weather factor as a "causative" instrument based on the specific historical data and not specific knowledge that typical weather forecasters have. The use of the bootstrap method to verify the indications of the VaR method is another advantage of the presented model. The obtained additional confidence interval is strengthening the VaR indications. The implementation of the weather VaR concept to derivative valuation may significantly influence the market of forward-looking weather contracts.
\end{abstract}

Keywords: weather risk, value-at-risk, bootstrap.

JEL Classifications:C100, C500, G100, G320

\section{Introduction}

As reported by Ch. Toeglhofer, R. Mestel and F. Prettenthaler (Toeglhoferetal., 2012) a weather risk assessment addresses two important issues: it indicates the economic impact of weather and climate changes and the degree of

DOI: $10.24818 / 18423264 / 54.1 .20 .03$ 
use of derivative weather instruments in reducing weather risks and an adaptive climate change strategy. Therefore, it is interesting to incorporate methods of measuring market risk into weather risk conditions. All attempts to indicate the scale of weather hazards with predetermined probabilities may be extremely important, both in the market for forward-looking weather contracts and in the general anticipation of events of specific weather factors. Regardless of which concept is considered, this has a strong influence on the economic nature of negative changes in the weather.

The use of the so-called compartmental methods, such as the Value-at-Risk methodology commonly used in the financial world or the bootstrap analysis, may be extremely useful here. However, the main goal of the authors is not to investigate the financial impact of unusual changes in weather conditions, but to indicate equally high effectiveness in predicting future weather states of the underlying instruments in relation to traditional methods of determining such types of forecasts. Thus, more complex issues, such as risk exposure, will be omitted.

The concept presented in the article is slightly different from the one discussed, e.g. in the work of F. Prettenthaler, J. Köberl and D. Neil Bird (Prettenthaleret al., 2016), although it could be called the Weather-VaR in the context of the Value-at-Risk or, Weather-bootstrap when one considers the bootstrap analysis. This type of concept also aims to show that the aforementioned methods (mainly VaR) are a flexible concept of modeling future meteorological states, which translates, among others, into a more precise valuation of weather derivatives or much more predictability of weather conditions in the near future. This allows both a more effective "fight" against weather hazards both in specific sectors of the economy and in the so-called crisis management process.

\section{Weather-VaR concept and methodology}

While looking in more detail at the weather risk management process, there is often a problem of lack of knowledge regarding not the impact of deviations from the norm, but the question of indicating the worst case scenario, i.e. estimating the maximum risk for investments in relation to the fluctuation of a given weather factor. These dilemmas are perfectly solved by the concept of value at risk, quite commonly used in the case of market risk.

Value-at-Risk $(\mathrm{VaR})$ in its structure is a measure of the maximum potential loss in a given period within a given confidence interval, substantially $95 \%$, which corresponds to the value of two standard deviations (figure 1). In other words, VaR is a measure that does not foresee the worst scenarios, but maximum losses, as long as the volatility of the underlying instrument remains within the limits of, say, the two $\sigma$. This measure is thus understood as the value of the respective percentile or, as someone would prefer, the cut-off point of the specific density function. It should be remembered that according to the VaR definition it refers to the left tail of the distribution. For example, in the context of an enterprise's income, taking into account annual periods, a loss with a probability of $5 \%$ will hypothetically 
Weather Risk Management in the Weather-VaR Approach. Assumptions of Value-at-Risk Modeling

occur once every twenty years. At the same time, the company receives information that in $95 \%$ such a loss will not be exceeded during this twenty-year period.

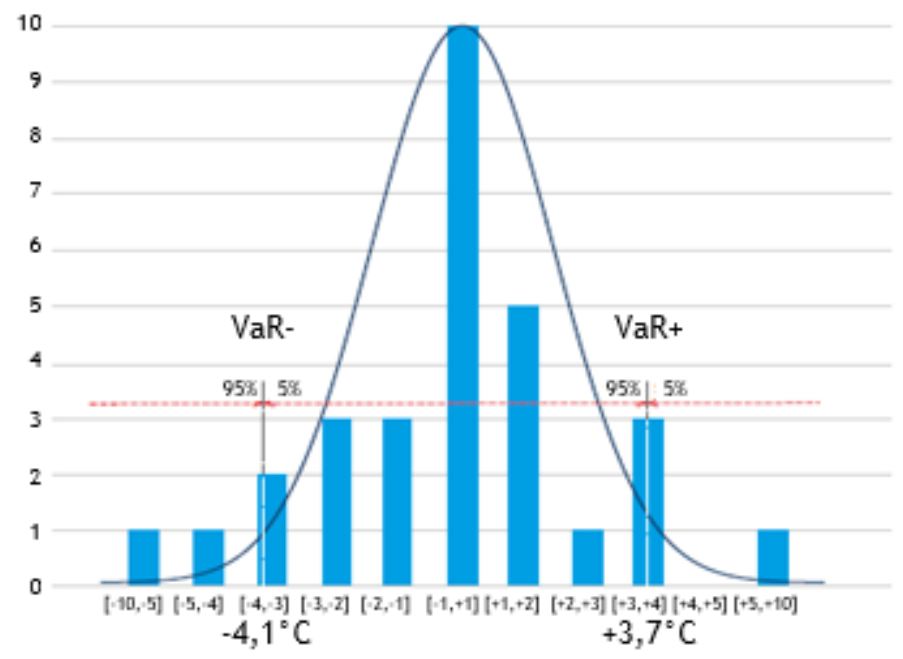

Figure 1. Schematic illustration of the Weather-VaR idea on the example of temperature changes - in autumn. Source: The authors' own research.

The implementation of Value-at-Risk in weather conditions contributes to the quantification of threats occurring on the side of nature. The concept, named Weather-VaR by authors, allows a better assessment of climate-related investments. Its essence is to transform unpredictable weather into one feasible, objective, easy to understand metric system. Weather value at risk is the focus on the variability of weather factors, defined as the observed deviation of weather conditions from its normal, long-term value. In the simplest terms, Weather-VaR is, to a large extent, an objective and quantitative method determining the value of potential maximum changes caused by the climate threat in a time horizon important for the entrepreneur and investor. Thanks to the identification of climate changes, this concept allows significantly mitigating the risk of weather in the short term, as well as implement appropriate business strategies to build long-term resilience to climate change (using financial instruments, as derivatives or weather insurance products).

Referring to the Value-at-Risk, one can define the weather risk itself as potential losses resulting from weather variability for a given period, within a certain confidence interval. The total weather risk is the same sum of all such types of risk, taking into account possible correlation between weather changes. TheWeather-VaRconnection with weather risk and the process of its management 
is, therefore, intuitive and can be illustrated using the diagram (Error! Reference source not found.).

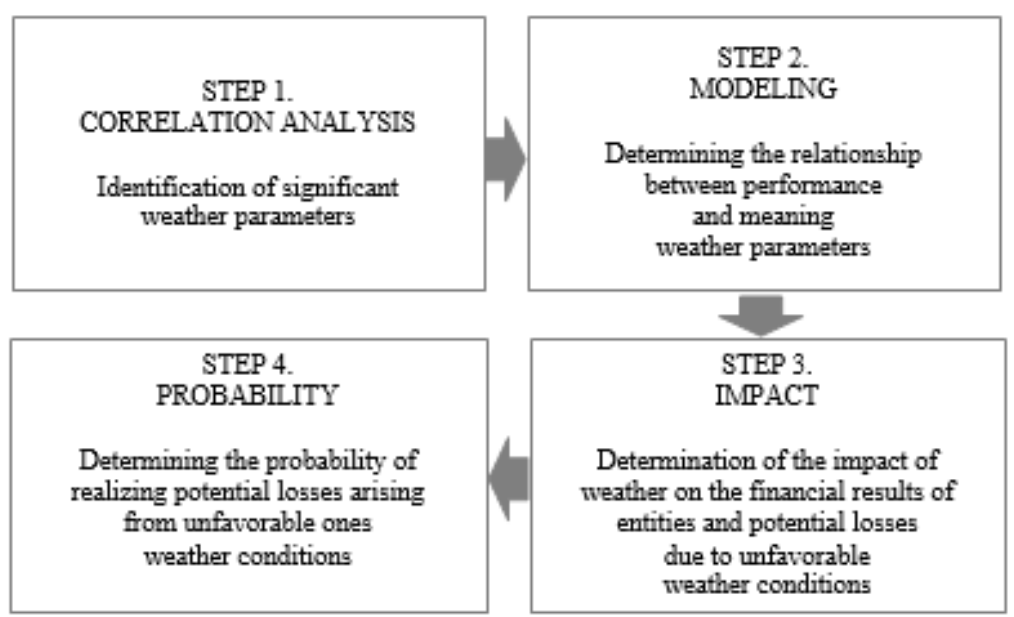

Figure 2. Diagram of the weather management process with regard to the WeatherVaR methodology.

Source: The authors' own research.

The first step of the process is creating a database of weather variables affecting the entity's operations and systematic cross-analysis of the company's data with weather variables. Such behavior is aimed at selecting weather factors that have a statistically significant impact on the results of a given company (for sale, profit, costs, etc.). In most cases, one or two weather variables are enough to capture the effect of weather on the subject.

The next stage refers to the modeling of relations between atmospheric variables distinguished in the first stage. It should be emphasized here that the relationship between weather and business activity is not always linear. There may also be threshold values for a given weather factor for which a given factor has an impact on the entity's financial result, and below or above which such impact is negligible. As in the first stage, we test different types of models to find relationships that best suit the company's performance.

The third stage is the analysis of historical data. In this case, an analysis is made in terms of the average and maximum loss caused by adverse weather conditions. The average loss is defined as the arithmetic mean of all negative effects in a given period, for example over a period of several years. In turn, the maximum loss refers to the most unfavorable weather conditions in the analyzed time horizon. The market risk measures developed by RiskMetrics TM can play a significant role here. This is mainly about the RiskGrades methodologies, such as Worst-Case Performance - WPC, Worst Losing Streak - WLS, eXpected Loss - XL, etc. (Mentel, 2012). 
Weather Risk Management in the Weather-VaR Approach. Assumptions of Value-at-Risk Modeling

The final stage of the process, defining the probabilities of realizing potential losses resulting from unfavorable weather conditions, is $\mathrm{VaR}$ modeling. Allows you to indicate the so-called value at risk at a predetermined level of probability. Valueat-risk is, however, a value applicable both in the third and in the fourth phase of the process, as it estimates the amount of potential losses that may occur and the scale of probability of their implementation.

Diagram 2 confirms a significant approximation of the methodology of weather risk management, with what is commonly used in the process of market risk management. Also in this case, companies must make attempts to systematically evaluate the methods used to achieve the appropriate priorities and to secure the allocation of stocks. Formally, this concept closes in five steps (Error! Reference source not found.).

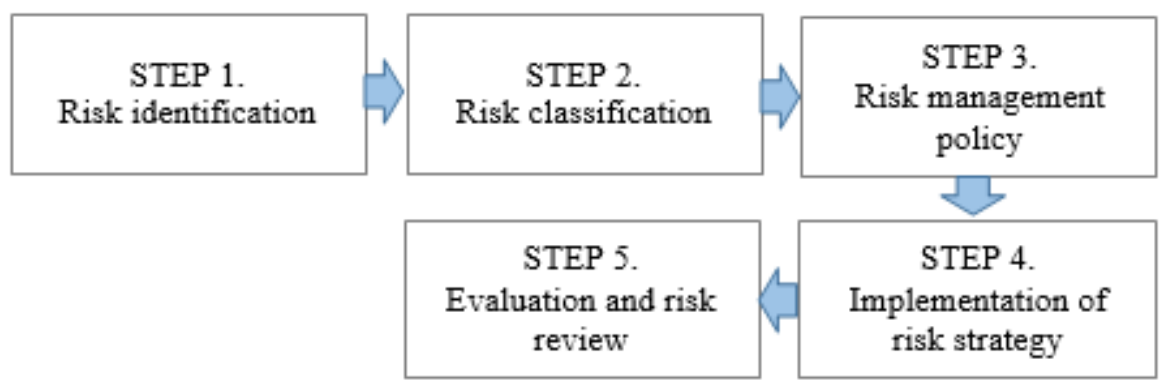

Figure 3. Weather Risk Management cycle. Source: The authors' own research.

Assuming that the Value-at-Risk methodology is an effective tool for weather risk management, a brief consideration should be given to the analysis of different modeling concepts of this magnitude. Not all methods of calculating it are equally effective. The possibility of estimating VaRin several ways is both an asset and an obstacle, as the diversity of approaches allows for the selection of better or worse concepts in this respect. On the other hand, the multiplicity of methods is not welcomed, the more so because many of them allow estimating the amount of potential losses using an advanced mathematical apparatus.

When making a fundamental separation between different VaR concepts, three groups should be distinguished. The first of them is a group based on models describing the „behawior” of the underlying instruments. This type of approach uses the assumption that the percentage changes of the analyzed instruments are characterized by a certain probability distribution. However, sometimes the assumption of this type is sometimes false, because it is often difficult to match the said distribution. Then, the second group of methods of estimating the value at risk, i.e. non-parametric methods, comes to help. They are a kind of alternative to more advanced concepts. Often this group is categorized as so-called simulation. The 
link between these groups is another class of semiparametric methods. In literature, it is often referred to as non-parametric methods, which is why it is not always distinguished as a separate category(Mentel, 2012).In addition, hybrid models are a combination of the aforementioned(Mentel, 2008).

When considering the parametric methods of estimating the value at risk, it should be emphasized that there are many analytical models describing the fluctuation of instruments. These models are mainly implemented by analysts and financial engineers. The basic premise allowing for their differentiation is a different approach to modeling random disturbances (e.g. Normal distribution, GED or Student's t) and the methodology of calculating the value at risk. One can distinguish a group of methods based on generalized autoregressive processes of conditional variance (for example, the GARCH type $(1,1)$ ), the "Mean Reversion" models or, for example, random walk (Random Walk). Of course, there are many more of these concepts.

The most commonly used model in this category is the classic concept of drift with random disturbances modeled using the normal distribution, commonly known as RiskMetrics Normal Drift(Mentel, 2012). In this model, so-called conditional variance for daily percentage changes of a given instrument (with the practical assumption that their average value is equal to zero) is estimated as an infinite moving average with exponential weights. The $V a R$ boundaries calculated on its basis (for the assumed significance level $\alpha$ ) for the one-day time horizon will be for certain weather factors (in this case for temperature) the form:

$$
T_{t-1} \cdot \exp \left(\mu+\sigma_{t} \cdot \tau_{N(0,1), \alpha / 2}\right) \leq T_{t} \leq T_{t-1} \cdot \exp \left(\mu+\tau_{N(0,1), 1-\alpha / 2} \cdot \sigma_{t}\right)
$$

where:

$\tau_{N(0,1), \alpha / 2}, \tau_{N(0,1), 1-\alpha / 2}$-respectively the quantiles of a given order in a normal distribution.

The appropriate model parameters are determined by the maximum likelihood method.

When characterizing the next group of simulation methods, it should be distinguished between historical simulation and Monte Carlo. The advantage of this category, considered by some to be quite significant, is that it gathers nonparametric methods. On the one hand, there are no limitations resulting from the necessity of assuming normality, on the other hand it avoids the estimation of some parameters based on historical data. The point here is, among others, values such as the mean or standard deviation.

The third listed group, the semiparametric methods, is based on the extreme value theory The Extreme Value Theory $(E V T)$, which deals with distributions having thick tails. According to this method, extreme observations come from a different distribution than the entire distribution of the analyzed variable. The EKT methods are often used in this approach (Emmer-Klüppelberg-Trüstedt) (Mentel, 2013), BM (Block Maxima) (Lindholm, 2015)and POT (Peaks-Over-Threshold) (Ghorbel, Trabelsi, 2008). Essentially, these approaches are based on the Hill 
Weather Risk Management in the Weather-VaR Approach. Assumptions of Value-at-Risk Modeling

estimator or, as in the case of the POT model, equally well on the assumption that the tail of the percentage change distribution comes from the generalized Pareto distribution.

\section{Value-at-Risk determinants}

When analyzing the issue of weather risk modeling using the VaRmethodology, attention should be paid to the determinants responsible for the estimates obtained as a result of its use and, as a result, its effectiveness. There are many issues that „proper consideration” significantly improves the quality of the indications we receive for Value-at-Risk.

An important element of the whole concept is the number of historical observations that should be taken into account in order to estimate the value of future losses. There is a necessity to determine the so-called the minimum number of observations necessary to estimate a standard deviation estimator for, for example, one-day returns of the value of the instrument under examination. Based on the dependencies developed by RiskMetrics ${ }^{\mathrm{TM}}$ in this case, one can use the dependency (Mentel, Brożyna, 2014):

$$
n=\frac{\ln \left(\gamma_{t o l}\right)}{\ln (\lambda)}
$$

where $0<\gamma_{t o l}<1$ it is a sufficiently low level of tolerance, and $\lambda$ is a smoothing constant.

Thus, assuming a smoothing constant at 0.97 with a significance level of 0.01 , we obtain $\mathrm{n}$ oscillating close to 150 (an accurate value of 151 observations is assumed then). However, if the tolerance level is set at 0.05 , the number of historical observations is significantly reduced to the level of $n$ equal to about 100 . Differences in estimates of the value at risk for the various number of historical observations included in its estimation are shown in figure 4.

Moving further towards analyzing the factors responsible for the effectiveness of future risk forecasts using $V a R$, it would be appropriate to classify individual concepts of value at risk into two groups, i.e. simulation and non-parametric methods, as is done by some scientists and practitioners.

With this approach it can be concluded that the first of these groups is characteristic because it does not assume any assumptions as to the form of the subject's distribution of the series, and moreover, the methods of deriving the variability do not use any equations. The variability index determines the change in the value of the weather factor corresponding to a quantile equal to the required level of confidence. Percentile methods, how often to name them, are preferred by those who believe that the assumption of normality is a weak point of the overall VaR model.

However, a significant drawback of these methods is the assumption that variability is constant at a given time, and thus these models attribute equal weight 
to every daily return. This is widely accepted. However, such variations are not permanent, on the contrary, they are almost constantly changing. In fact, the data show irregular but often abrupt changes in volatility, i.e. after a period of low volatility, there is a period of high volatility.

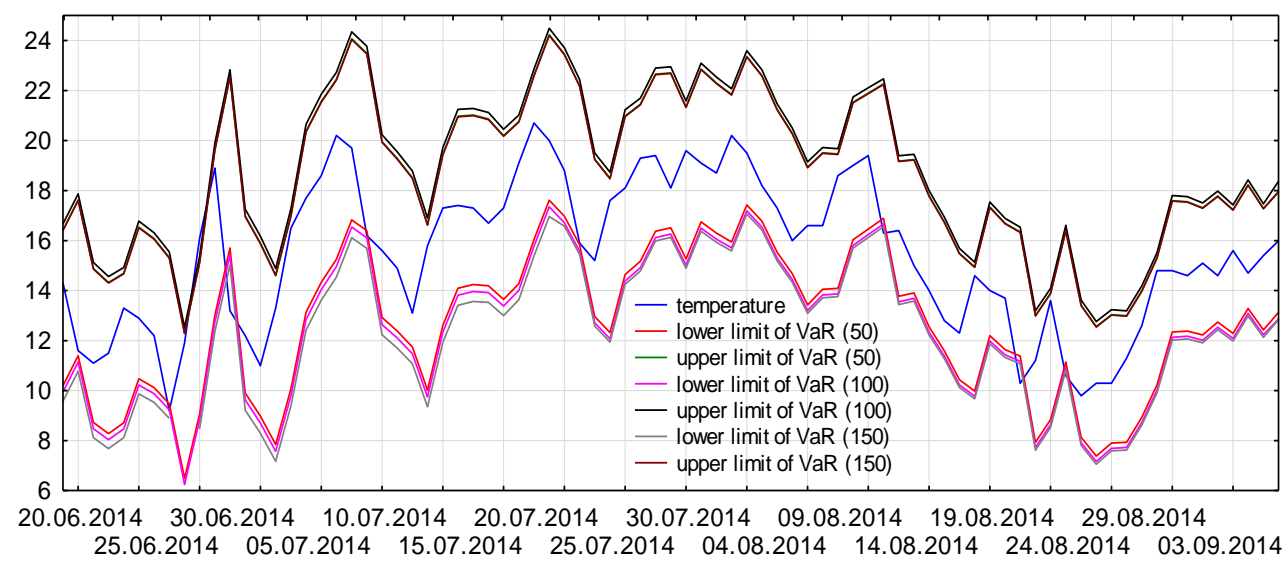

Figure 4. Sample Value-at-Risk estimates using the Monte Carlo simulation method (lower and upper limits) for temperature in the Malopolskie Voivodeship for different amounts of historical observations included in the study.

Source: The authors' own research.

Such an approach implies the necessity of using other models, and the explanation of this state of affairs is a phenomenon defined in the world of finance as a grouping of income from financial assets, where the information immediately affects the situation on a given day, and to a lesser extent what is happening in the next days, i.e. the impact „breaks down” in a fairly short time. The occurrence of autocorrelation causes that the data from the last period provide more information about the current level of volatility than those from the previous period. The suggestion is that to obtain a volatility model, which precisely measures its current level, it would be necessary to assign higher weights to the last values (Mentel, Brożyna, 2015).

This is also the case of the second group of models, which includes analytical models that describe the "behavior" of the instruments in question in various ways. One can distinguish the models developed by the group of J.P. Morgan, based on volatility models created using the exponentially weighted moving average (EWMA) (Crowder, 1987; Mentel, 2012)and the GARCH models (generalised autoregressive conditional heteroskedastic) (Bollersev, 1986).

EWMA is an important element of the VaR model known as RiskMetrics ${ }^{\mathrm{TM}}$ (fig. 5). The equations for deriving these variations are described as:

$$
\sigma_{t}^{2}=(1-\lambda) r_{t}^{2}+\lambda \sigma_{t-1}^{2}
$$

and 
Weather Risk Management in the Weather-VaR Approach. Assumptions of Value-at-Risk Modeling

$$
\sigma_{t}^{2}=\alpha_{0}+\alpha_{1} \cdot r_{t-1}^{2}+\beta_{1} \cdot \sigma_{t-1}^{2} ; \alpha_{0}, \alpha_{1}, \beta_{1}>0
$$

While in the EWMA model the parameters are simple to estimate, in GARCH class models (in many varieties) their determination is not always an easy process. Their estimation requires the maximization of the credibility function. The occurrence of extreme changes in many data here may cause problems for the function of maximum likelihood - used to calculate parameters manifested in the lack of convergence.

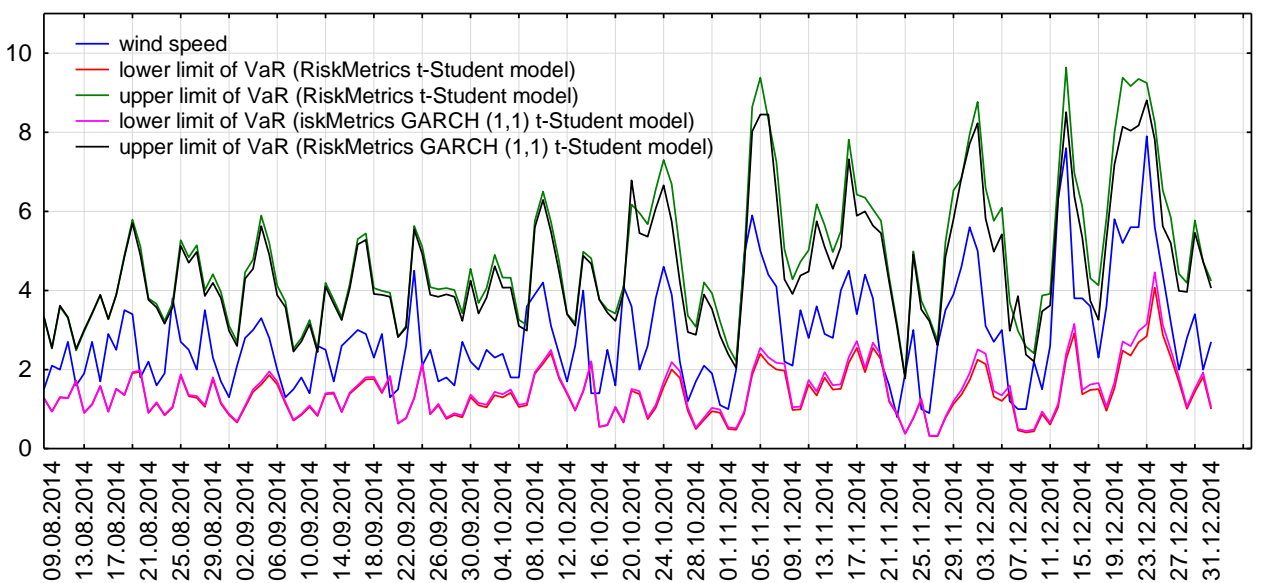

Figure 5. Sample Value-at-Risk estimates (lower and upper limits) for wind speed in the PodkarpackieVoivodeship for selected models of the EWMA and GARCH classes. Source: The authors' own research.

The main difference between the EWMA model and the GARCH class models is the fact that the second group of models responds even more aggressively to changes in the time series than the EWMA model. In addition, it seems that the interesting and useful feature of the GARCH models is that they include the phenomenon of „return to medium”. This is mainly due to the fact that the value of some financial assets fluctuates around a certain long-term value.

An important differentiating factor for the VaR methodology is the assumed level of significance. Its appropriate value allows obtaining both reliable indications using this method and to keep an appropriate scale of confidence in the estimated results. Reducing the level of $\alpha$ gives you a bigger so-called predictability, however, often entails significant over-estimation of possible threats, which unnecessarily contributes even to increased risk aversion and disturbances in real changes in weather factors. In turn, the action consisting in increasing the value of $\alpha$ gives a much better fit to the actual changes, but the certainty as to the correctness of these estimates is much smaller (fig. 6). 


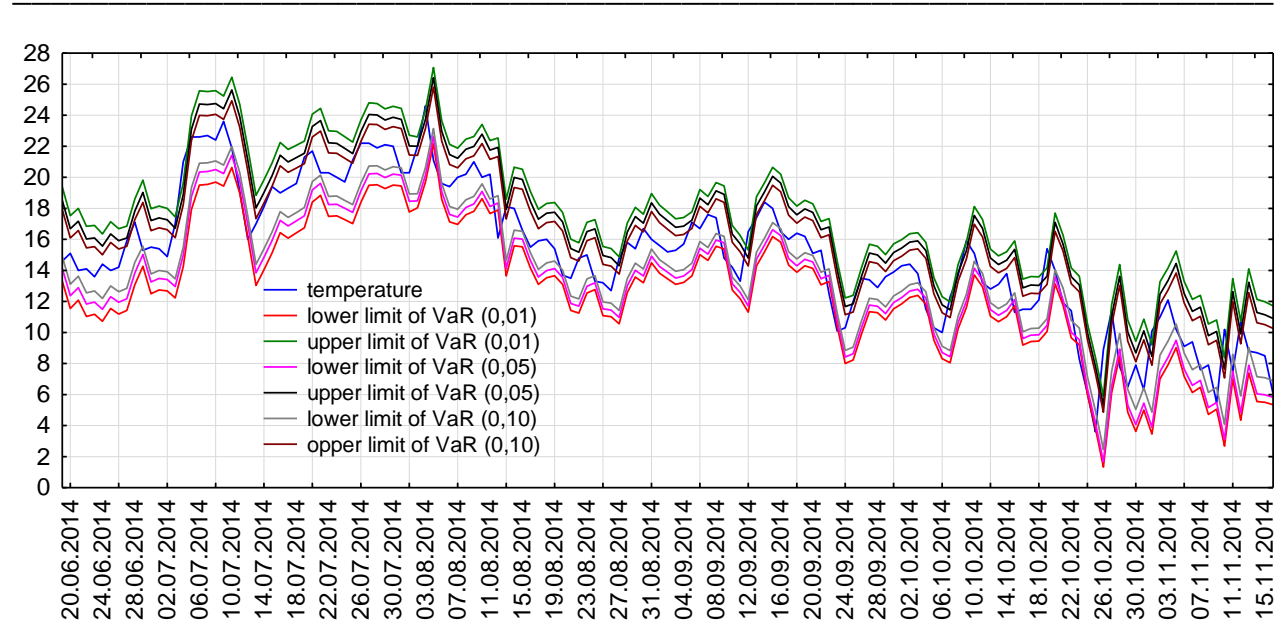

Figure 6. Sample Value-at-Risk estimates (lower and upper limits) for temperature in the West Pomeranian Voivodeship receive using the RiskMetrics Normal model, taking into account different levels of significance. Source: The authors' own research.

It should be emphasized that there are methods that are characterized by better effectiveness at low values of significance level, and models that are more effective when $\alpha$ is slightly higher. A typical example of this is the application of a concept in which random disturbances are modeled using generalized error distribution (General Error Distribution - GED). Due to the specificity of this distribution, i.e. the fat tail thickness, is often used in practice. It can be said that this is one of the few concepts of value-at-risk approach that improves its value as the level of significance decreases. Most of the methods used behave the other way around, because in their case the overall precision of the models increases with the value of the significance level adopted for analyzes (Mentel, 2011).

The mentioned modeling of random disturbances is of considerable importance in the whole process. Diversity in this area is formally limited to two typical concepts, namely the case in which this type of disturbance is modeled either by normal distribution or by student's $t$-distribution. Other variants are less common, but they do not mean they are not used. It is enough to refer to the already mentioned GED concept.

Different approaches to random error modeling differentiate $V a R$ estimates. Even with the same model, however, with a different way of modeling the mentioned disturbances, slightly different indications are obtained. These estimates may not be diametrically different, but often affect the final evaluation of the effectiveness of a given method, improving or degrading the results (fig.7). 
Weather Risk Management in the Weather-VaR Approach. Assumptions of Value-at-Risk Modeling

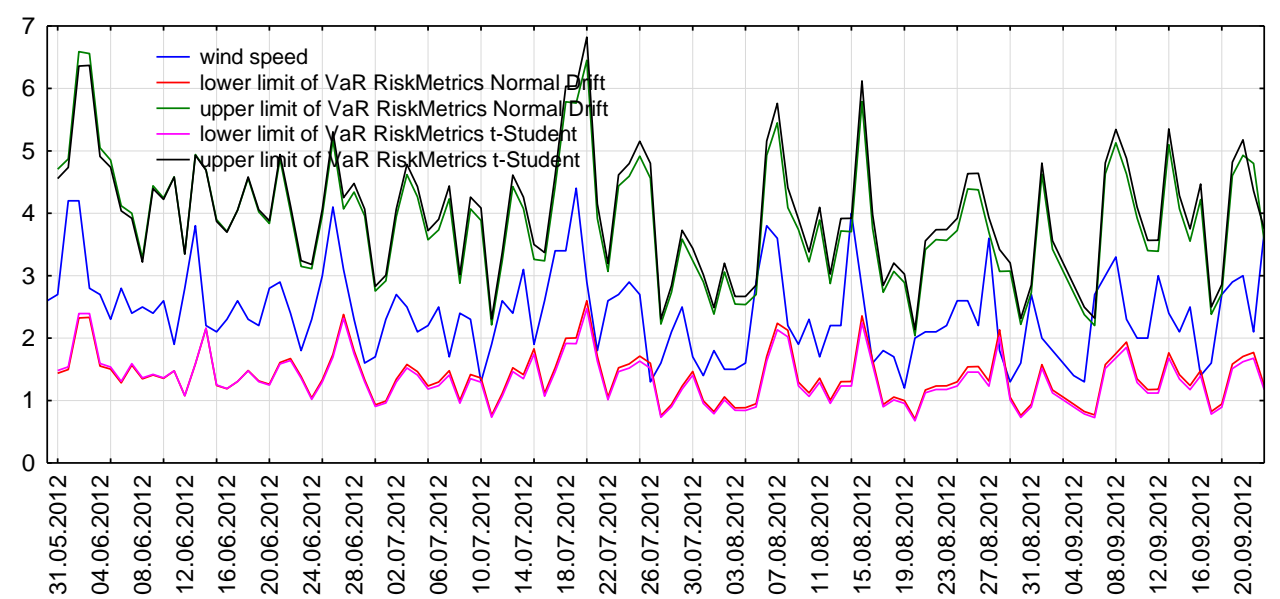

Figure 7. Sample Value-at-Risk estimates (lower and upper limits) for wind speed in the West Pomeranian Voivodeship receive using the classical RiskMetrics model taking into account various concepts of random disruption modeling. Source: The authors' own research.

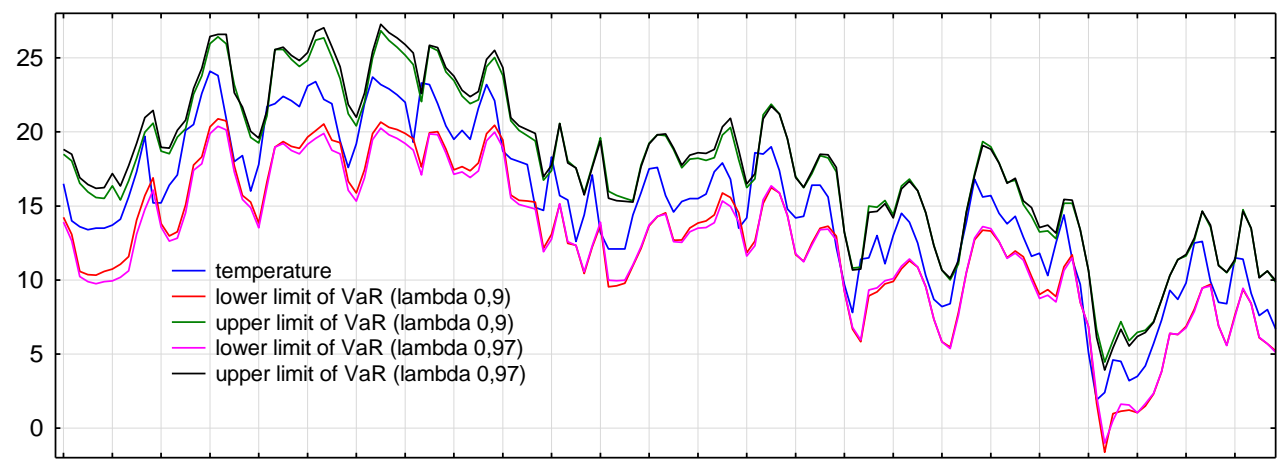

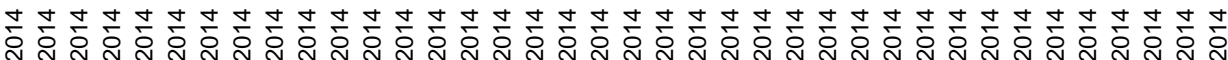
фi छ

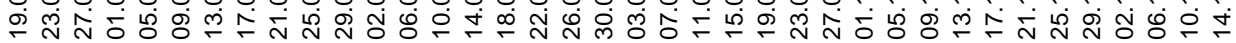

Figure 8. Sample Value-at-Risk estimates (lower and upper limits) for temperature in the Lodz Voivodeship receive using the classical RiskMetrics Normal model, taking into account different smoothing constant values $\lambda$. Source: The authors' own research.

Returning to derivation of variability, one should also consider constant smoothing in models in which it is created by means of the previously analyzed exponentially weighted moving average. In the EWMA class models, the value of $\lambda$ is significant (fig. 8). This element is treated as so-called the aging factor of information and should be differentiated due to the nature of the data. It is 
important whether the data is daily or monthly or represent other periods. A higher level $\lambda$ allows to obtain a medium variation from a longer period and a correspondingly lower index $\lambda$ allows one to approximate the variability for shorter periods. For example, for one-day periods, the volatility measure takes into account the significant and rapid aging of information; is therefore based on recent observations in the historical series. However, it gives a better fit to real data (Mentel, Brożyna, 2015).

\section{Long-term forecasting}

When characterizing the concept of value-at-risk in the context of modeling weather factors, it is worth paying attention to one important asset, which in this case may prove to be useful. The main purpose of the value at risk is to indicate future states of a given instrument. As the forecasts presented so far were for oneday periods, it should be tempted to generate weather forecasts for periods that are much ahead.

With appropriate rescaling, commonly used VaR models bring about the generation of long-term forecasts. Then the modeled variability is expressed as follows:

$$
\begin{aligned}
& \sigma_{t+h \mid t}^{2}=h \cdot \sigma_{t+1 \mid t}^{2} \\
& \sigma_{t+h \mid t}=\sqrt{h} \cdot \sigma_{t+1 \mid t}
\end{aligned}
$$

The resulting ,forecast cones" are a consequence of the mentioned long-term variance scaling with the forecast horizon $h$ (fig. 9). Perhaps they do not give very reliable indications about future values, for example rainfall or temperature, but to a large extent indicate a trend of change. In certain situations, when it comes to the risk of weather changes, long-term VaR allows greater predictability of the effects of your investments and activities.

By transferring the Value-at-Risk to weather risk management, it is impossible to ignore it based on the so-called profit and loss(Mentel, 2012). In such a variant, the potential values of the underlying instruments are initially calculated in the period $t$ from the formula:

$$
T_{t}=T_{0} \cdot e^{r \sqrt{t}}
$$

where:

$T_{t}$ - temperature value in $t$ period,

$T_{0}$ - temperature value in the initial period,

$r$ - the rate of return previously determined,

$t$ - the time horizon for which $V a R$ is determined,

and then differences are generated between the value of, for example, temperature $T_{0}$ and its scenarios $T_{t}$. The differences obtained as a result of this process are then subjected to the ordering process, and only then the right percentile corresponding to the assumed confidence level is determined (fig. 10). 
Weather Risk Management in the Weather-VaR Approach. Assumptions of Value-at-Risk Modeling

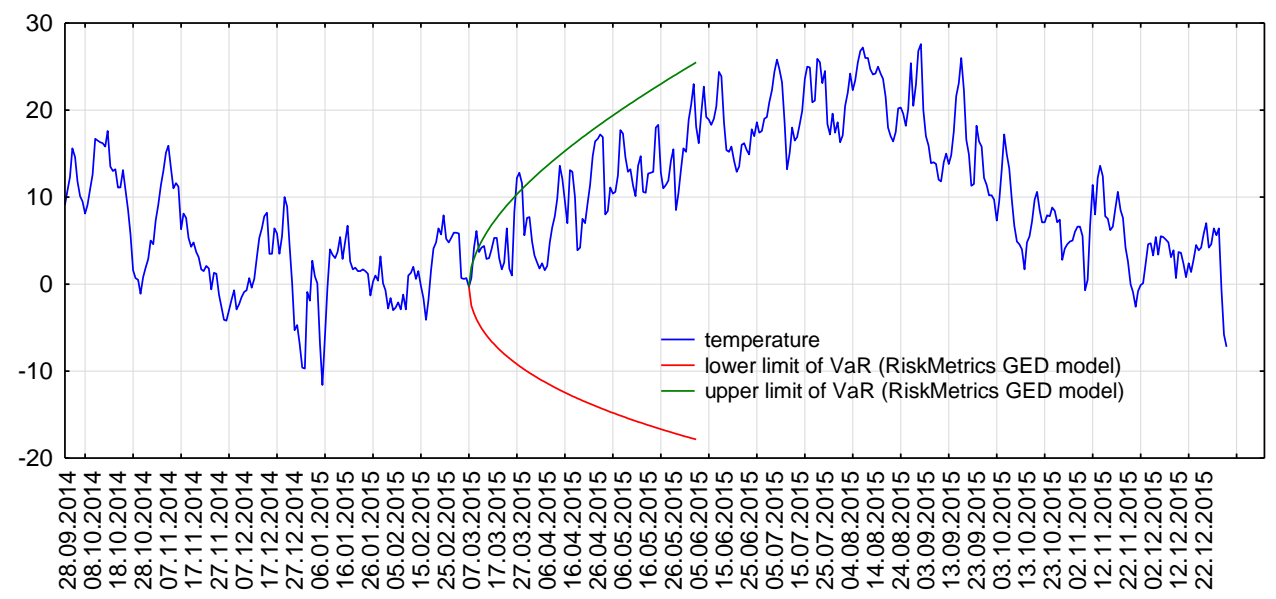

Figure 9. Sample of a long-term forecast (RiskMetrics GED model) for daily temperature values in Rzeszow $(\alpha=0,05 ; h=90)$.

Source: The authors' own research.

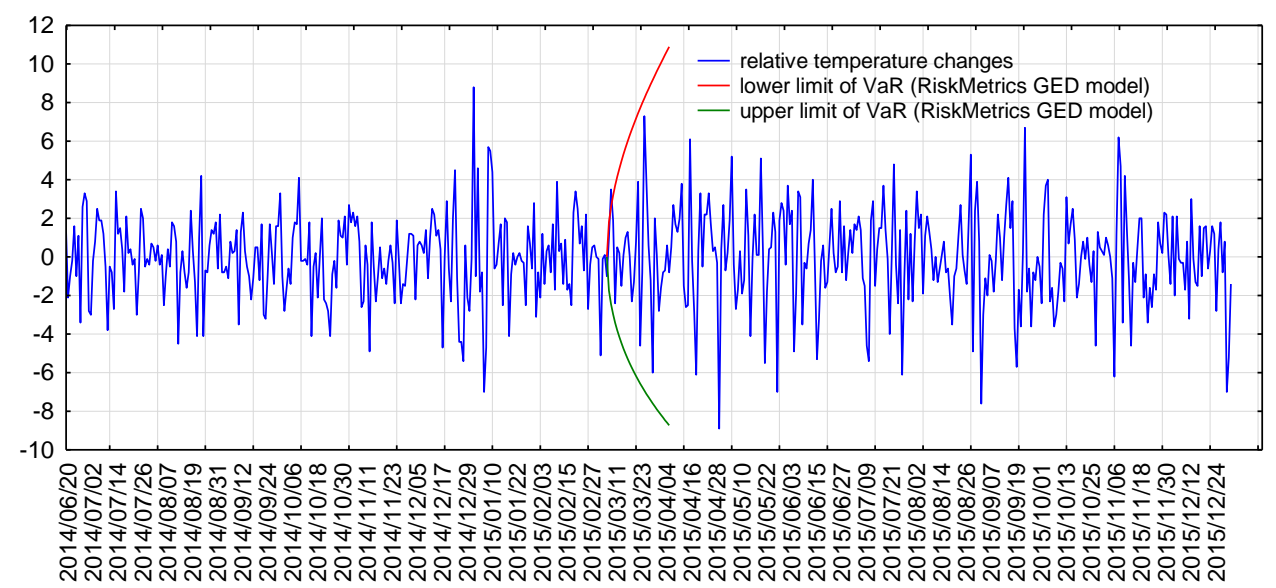

Figure 10. Sample of a long-term forecast (RiskMetrics GED model) for daily relative temperature changes in Rzeszow $(\alpha=0,05 ; h=30)$. Source: The authors' own research.

\section{Bootstrap as a control over the Value-at-Risk error}

When analyzing estimates of Value-at-Risk in the context of weather time series, it is worth strengthening the effectiveness of predictions by implementing the bootstrap method. It is especially useful in conditions where the distribution of a given feature is not known.

Apart from the issue of the increasing use of bootstrap methods in econometrics as an alternative tool in quantitative analysis, the more valuable is the 
use of the so-called concept. sampling to gain control over the error rate in the VaR weather calculations made.

Referring to the interpretation of such methods, it should be noted that they rely on a random sampling of samples in order to later use them to estimate the bootstrap values of various functions. Over the years, they have gained importance, thus finding application in many fields of science. Efron is considered the precursor of their implementation (Efron, 1979), who used them for statistical inference. It was a breakthrough moment and from now on the importance of this type of method is constantly increasing.

Returning to the essence of simulation approaches, it is necessary to emphasize twofold application. First of all, the main idea behind the use of bootstrap is to design a Monte Carlo experiment that allows one to generate a distribution of the statistics considered at a given moment based on data from a single sample(Efron, 1979). Secondly, an application of the discussed methodology is drawn in the analysis of time series (Hall, 1992). In this case, they are used to analyze the structure of such a series or to verify the hypothesis assuming the form or government of integrating the series. They are also often used in testing the power of tests, because they provide a more accurate asymptotic approximation compared to the Monte Carlo simulation.

While limiting the considerations to the time series, one should also emphasize the significant diversity in this case. The most commonly used bootstrap algorithms are: block bootstrap (Radovanov, Marcikić, 2014),sieve bootstrap(Buhlmann, 1997),wild bootstrap (Liu, 1988),pairs bootstrap(Freedman, 1981), recursive bootstrap (Ogland, 2014),stationary bootstrap(Politis, Romano, 1994).

Although there are several items listed, these are not all algorithms used in this field. They are different, including the so-called hybrid approach. Bootstrap methods have also gone deeply into financial analysis. Examples are presented, for example, in the works ofH. Li and G.S. Maddala (Li, Maddala, 1996)or J.L. Horowitz(Horowitz, 2001). Using these methods, an attempt was made to determine the density of so-called predictors of percentage changes in prices or their ranges for various models (Thombs, Schucany, 1990)and for estimating the Value-at-Risk(Vlaar, 2000).

Due to the many different concepts of bootstrap methods(Domański, Pruska, 2000)in order to illustrate their use in controlling the VaR error rate, the concept described by J. Leśkow, J. Mokrzycka and K. Krawiec (Leśkowet al., 2011) was used.The presented approach is interesting because it was successfully used in the financial time series, from where the concept of Value-at-Risk is derived. Thus, the implementation of the risk value and the bootstrap methods in weather conditions turns out to be extremely interesting. Additional compartments that were obtained as a result of the so-called of sampling, they constitute a strengthening of VaR indications.

In order to determine the exemplary confidence interval for $V a R$, the value at risk was initially estimated, based on daily temperature changes („temperature 
Weather Risk Management in the Weather-VaR Approach. Assumptions of Value-at-Risk Modeling

gains and losses") for the city of Rzeszów. The estimation of the value at risk was carried out using the GARCH model (1.1) with random disturbances modeled by the t-Student distribution.

On the basis of the so-estimated risk value, the confidence intervals were determined. In this case, the nonparametric bootstrap method was used (Lahiri, 2003), which the work (Leśkowet al., 2011)refers to.

The sampling was carried out on the generated residues $\left(\varepsilon_{l}, \ldots, \varepsilon_{n}\right)$. Verification of their independence was performed on the basis of autocorrelation function analysis (ACF) and sample autocorrelation (PACF). Thanks to the conducted sampling, bootstrap observations $\left(\varepsilon_{1}^{p}, \ldots, \varepsilon_{n}^{p}\right)$ were created, which in turn contributed to the determination of subsequent implementations of returns as:

$$
r_{t}^{b}=\mu+\sigma_{t} \varepsilon_{t}^{b}
$$

Therefore, it should be assumed that the empirical quantile of the temperature change function for returns is in this case a bootstrap estimator of the risk value, which can be written as $V \widehat{a R(} \alpha)^{b}$. The estimators obtained were used to estimate the confidence intervals for the value at risk. The confidence interval $\widehat{\operatorname{VRR}(\alpha)}$ at the assumed significance level $\beta$ is the interval $\left(u_{\frac{\beta}{2}}, u_{1-\frac{\beta}{2}}\right)$. The empirical quantiles in this case are respectively about $\frac{\beta}{2}$ and $1-\frac{\beta}{2}$ from the estimator distribution $\widehat{\operatorname{VaR}(\alpha)^{b}}$.

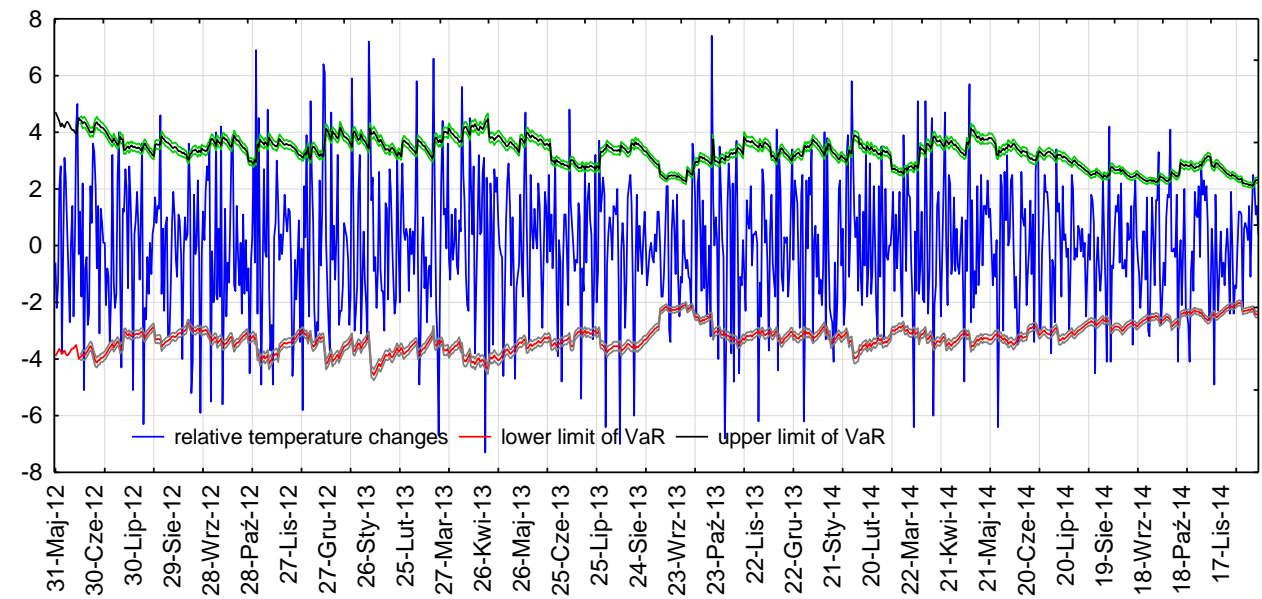

Figure $11.90 \%$ confidence interval for VaR for daily temperature changes for Rzeszow.

Source: The authors' own research.

An analogous procedure was carried out for the upper limit of VaR. Figures 11 and 12 illustrate the advantage of using bootstrap methods, which is the 
determination of the confidence interval for a value at risk. As a standard, the $95 \%$ confidence interval is wider than $90 \%$.

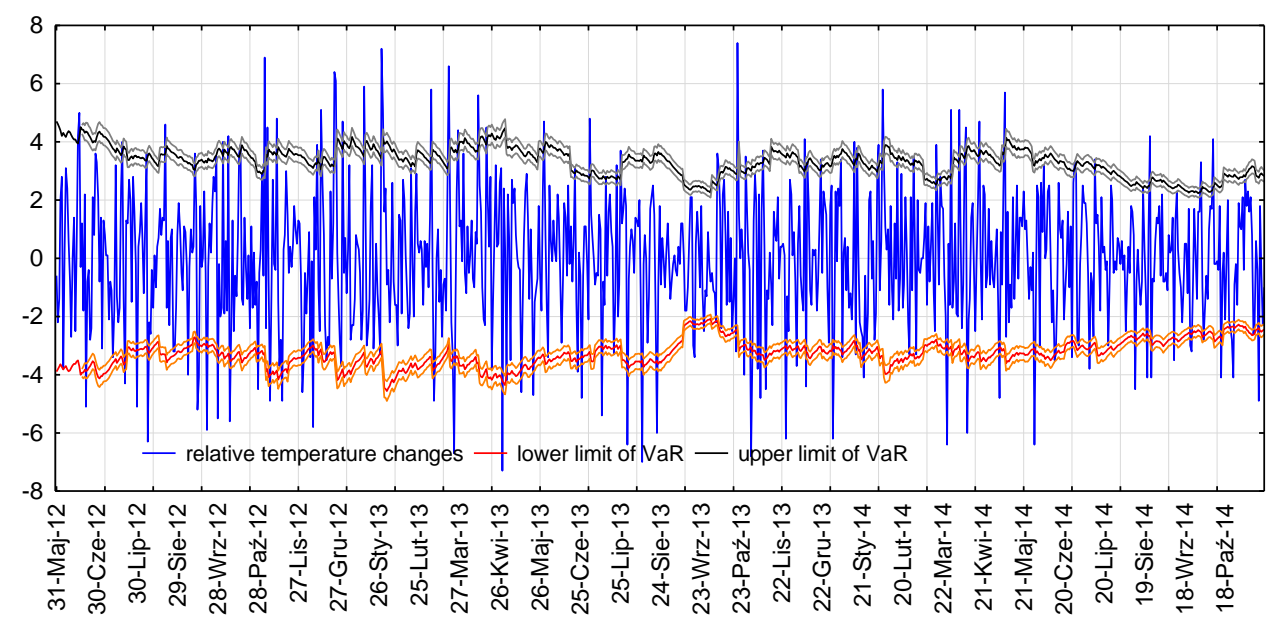

Figure 12. 95\% confidence interval for VaR for daily temperature changes for Rzeszow.

Source: The authors' own research.

\section{Conclusion}

The article is a proposal to increase the predictability of certain important weather factors by implementing a typical concept in the market risk analysis, $\mathrm{VaR}$, in weather conditions. Remember that the weather forecast is the approximate and most probable picture of the future state of the atmosphere. In addition, it is developed on the basis of what is now, what has already been and knowledge of the laws of nature, which give some suggestions as to what will be. So if average users are not able to fathom in more detail current states, they only have an approximate picture, and do not know all the laws and mechanisms governing nature, estimation of future levels of weather factors using methods used by specialists in this area, rather impossible. This Weather-VaR proposal is therefore an alternative in this respect.

When summarizing the Weather-VaR concept described, you should always consider its strengths and weaknesses. This concept:

- determines the probability of the change in the value of the weather factor,

- gives clear information about the predicted condition of a given weather factor,

- allows to a large extent in its forecasting function to increase the accuracy of valuation of weather derivatives, provided that we treat a given weather factor as the underlying instrument,

- gives greater certainty in the case of concluding weather contracts, 
Weather Risk Management in the Weather-VaR Approach. Assumptions of Value-at-Risk Modeling

- expresses the risk in a relatively easy way to interpret, and also allows its limiting,

- thanks to the capture of compartmentality allows a kind of „dominion” over weather changes and to a large extent to limiting financial losses,

- allows to determine certain trends in changes in weather factors by the possibility of determining „forecast cones”,

- enables enhancement of indications by, for example, applying an additional bootstrap analysis,

- is part of the concept of weather risk management process,

- is widely used and universal.

Referring to the disadvantages, the following should be mentioned:

- does not give an answer to the question, what changes I will make outside of its borders, i.e. what will happen if its value is exceeded,

- the results of the estimates are sensitive to the estimation method,

- estimates of parameters of the GARCH class models are not always an easy process; occurrence of extreme changes in many data may generate problems for the function of the highest reliability - used to calculate parameters manifested in the lack of convergence,

- it is impossible to use for daily amounts of atmospheric precipitation, because this value in such periods of time is in some sense a dichotomous variable,

- seasonality in longer periods precludes its use.

\section{REFERENCES}

[1] Bollersev, T. (1986), Generalized Autoregressive Conditional Heteroscedasticity. Journal of Econometrics (31);

[2] Buhlmann, P. (1997), Sieve Bootstrap for Time Series. Bernoulli, 3(2), 123-148;

[3] Crowder, S. (1987), Run-Lenght Distributions of EWMA Charts. Technometrics (29);

[4] Domański, C., Pruska, K. (2000), Nieklasyczne metody statystyczne. Warszawa: PWE;

[5] Efron, B. (1979), Bootstrap Methods: Another Look at the Jacknife. Annals of Statistics (7), 1-26;

[6] Freedman, D. A. (1981), Bootstrapping Regression Models. Annals of Statistics(9), 1218-1228;

[7] Ghorbel, A., Trabelsi, A. (2008), Predictive Performance of Conditional Extreme Value Theory in Value-at-Risk Estimation. International Journal of Monetary Economics and Finance, 2(1), 121-148. doi:DOI: 10.1504/IJMEF.2008.019218

[8] Hall, P. (1992), The Bootstrap and Edgeworth Expansion. New York: Springer Verlag; 
Yuriy Bilan, Grzegorz Mentel, Dalia Streimikiene, Beata Szetela

[9] Horowitz, J. L. (2001), The Bootstrap and Hypothesis Tests in Econometrics. Journal of Econometrics (100), 37-40;

[10] Lahiri, S. N. (2003), Resampling Methods for Dependent Data. Springer Series in Statistics;

[11] Leśkow, J., Mokrzycka, J., Krawiec, K. (2011), Zastosowanie funkcji kopuli w modelowaniu indeksów gieldowych. Finansowy Kwartalnik Internetowy e-Finanse, 7(2), 1-18;

[12] Li, H., Maddala, G. S. (1996), Bootstrapping Time Series Models. Econometric Reviews (15), 115-158;

[13] Lindholm, D. (2015), On Value-at-Risk and the More Extreme. A Study on Quantitative Market Risk Measurements. Uppsala: Uppsala University;

[14] Liu, R. Y. (1988), Bootstrap Procedure under Some Non-I.I.D. Models. Annals of Statistics (16), 1696-1708;

[15] Mentel, G. (2008),Hybrid Concepts of Long-Term Estimates for Value at Risk. Folia Oeconomica Stetinensia, 7(1), 1-12. doi:https://doi.org/10.2478/v10031-008-0004-0;

[16] Mentel, G. (2012), Ryzyko rynku akcji. Warszawa: Wydawnictwa Fachowe $\mathrm{CeDeWu}$;

[17] Mentel, G. (2013), Parametric or Non-Parametric Estimation of Value-atRisk. International Journal of Business and Management, 8(11), 103-112;

[18] Mentel, G., Brożyna, J. (2015), Decay Factor as a Determinant of Forecasting Models. International Journal of Economics and Finance, 7(1), 118-128. doi:10.5539/ijef.v7n1p118;

[19] Ogland, P. (2014), Mechanism Design for Total Quality Management. Using the Bootstrap Algorithm for Changing the Control Game. North Carolina: Lulu Press;

[20] Politis, D.N., Romano, J. P. (1994), The Stationary Bootstrap. Journal of the American Statistical Association, 89(428), 1303-1313. doi:10.2307/2290993;

[21] Prettenthaler, F., Koberla, J., Bird, D. (2016), 'Weather Value at Risk': A Uniform Approach to Describe and Compare Sectoral Income Risks from Climate Change. Science of the Total Environment(543), 1010-1018. doi:http://dx.doi.org/10.1016/j.scitotenv.2015.04.035;

[22] Radovanov, B., Marcikić, A. (2014), A Comparison of four Different Block Bootstrap Methods. Croatian Operational Research Review (5(2014)), 189-202;

[23] Thombs, L.A., Schucany, W.R. (1990),Bootstrap Prediction Intervals for Autoregressions. Journal of the American Statistical Association (85), 486492;

[24] Toeglhofer, C., Mestel, R., Prettenthaler, F. (2012), Weather Value at Risk: On the Measurement Noncatastrophic Weather Risk. Weather, Climate and Society, Vol. 4, 190-199;

[25] Vlaar, P. (2000), Value at risk models for Dutch bond portfolios. Journal of Banking \& Finance (24), 1131-1154. 